\title{
Constraints on Stellar Hydrodynamics from Abundance Anomalies of LiBeB and Metals
}

\author{
Georges Michaud $^{1}$, Jacques Richer ${ }^{1}$ and Olivier Richard ${ }^{1}$ \\ Département de physique, Université de Montréal, Montréal, Canada, \\ H3C $3 J^{7}$
}

\begin{abstract}
The availability of large atomic data bases has made it possible to calculate stellar evolution models taking into detailed account the atomic diffusion of all important contributors to opacity. The radiative accelerations and the opacity are continuously calculated during evolution taking the abundance changes of 28 species into account. This leads to the first self-consistent stellar evolution models for $\mathrm{A}$ and $\mathrm{F}$ stars. In $A$ and $F$ stars an iron-peak convection zone appears.

The calculated abundance anomalies are very similar to those observed in AmFm stars in open clusters except that they are larger by a factor of about 3 . To reduce the calculated anomalies to the observed ones, an additional source of turbulence (or some other hydrodynamical process) must be introduced. The mixed zone must extend about 5 times deeper than the iron convection zone. Detailed comparisons to a few AmFm stars have been carried out.

The LiBeB abundances observed in clusters give additional information. The abundances of the 28 species offer considerable constraints on the models. Various potential turbulence models have been introduced in a stellar evolution code and results of evolutionary calculations for $\mathrm{Li}$ gap stars are discussed in the light of the constraints offered by the abundances of $\mathrm{LiBeB}$ and metals. The radiative accelerations of $\mathrm{LiBeB}$ have also been recalculated taking the effect of changing metal abundances into account. This modifies the expected Li gap in the absence of turbulence.
\end{abstract}

\section{Introduction}

Helioseismology results show that atomic diffusion is occurring in the sun and suggest that some weak turbulence is also needed in order to account for the small Li abundance (Proffitt \& Michaud 1991; Chaboyer et al. 1995; Richard et al. 1996; Vauclair this conference). The presence of some turbulence Vauclair (1988) is also suggested by the progressive decrease of the Li abundance with the age of clusters as discussed elsewhere in this conference. Helioseismology results also show that, below the convection zone, the sun is rotating nearly as a solid body. This cannot be explained by any model yet suggested for the

\footnotetext{
${ }^{1}$ CEntre de Recherche en Calcul Appliqué (CERCA), 5160 boul. Décarie, bureau 400,
} Montréal, PQ, CANADA H3X 2H9 
turbulent transport of angular momentum. Taken together these facts show that while turbulence is probably an important particle transport process, none of the suggested models passes the test of the constraints imposed by helioseismology. The origin of turbulence in stars remains unknown.

Our approach is first to calculate stellar evolution models with all the physics of particle transport that is known from first principles. In radiative zones this implies atomic diffusion including gravitational settling, thermal diffusion and radiative accelerations, in addition to the purely diffusive term. We call these models the basic models. In a second step, simple turbulence models are introduced in order to better reproduce the observed surface abundances. Other hydrodynamical processes, such as mass loss or magnetic fields, could also be important but they will not be discussed here.

In this paper, results of stellar evolution are first presented for the basic models. This leads to the development of iron convection zones in stars of $1.5 M_{\odot}$ and larger and increases the depth of the convection zone in stars of 1.3 to $1.5 M_{\odot}$. Turbulence is then introduced in order to reduce the calculated anomalies in the basic models of A stars to the observed abundance anomalies in AmFm stars. The LiBeB observations will be used to constrain the turbulence in $F$ and $G$ stars and a summary of the constraints abundance anomalies put on turbulence in main sequence stars will be presented.

\section{Basic models and iron convection zones}

The basic models include the physics generally found in standard stellar evolution models, with the addition of atomic diffusion, including gravitational settling, thermal diffusion, radiative accelerations and the diffusive term. The detailed treatment of diffusion transport is described in (Turcotte et al. 1998). The largest contribution comes from the drift part of the diffusion velocity whose two most important terms are gravity and the opposing radiative acceleration:

$$
v_{g} \propto A g_{\mathrm{rad}}-\left(A-\frac{Z}{2}-\frac{1}{2}\right) g .
$$

When $g_{\mathrm{rad}}>g$, the involved element diffuses upward. A precise determination of radiative accelerations is essential; these are treated as described in Richer et al. (1998). For each atomic species (28), at each zone in the star (1000-1500), at each time step (500-1000 for an evolutionary sequence) integrals are carried out, at four points of the OPAL grid, over the spectral form $\left(10^{4}\right.$ frequency points) of the OPAL data. The Rosseland mean opacities are simultaneously recalculated. The OPAL data contains more than one gigabyte of data most of which must be in core memory during the calculations.

Furthermore, for stellar evolution calculations, it is in practice required to use the Burgers (Burgers 1969) formulation of the calculations of diffusion velocities since the hydrogen and helium contributions to momentum exchange with metals are of the same order. For the transport velocities, one ends up solving $56(=2 \times 28)$ coupled, non-linear, highly stiff, differential equations.

On one SGI R-12000 processor, it takes us less than 30 minutes to calculate the evolution of a typical standard model. However it takes some 3-5 days on four such processors to calculate the evolution of one of our basic models. More 

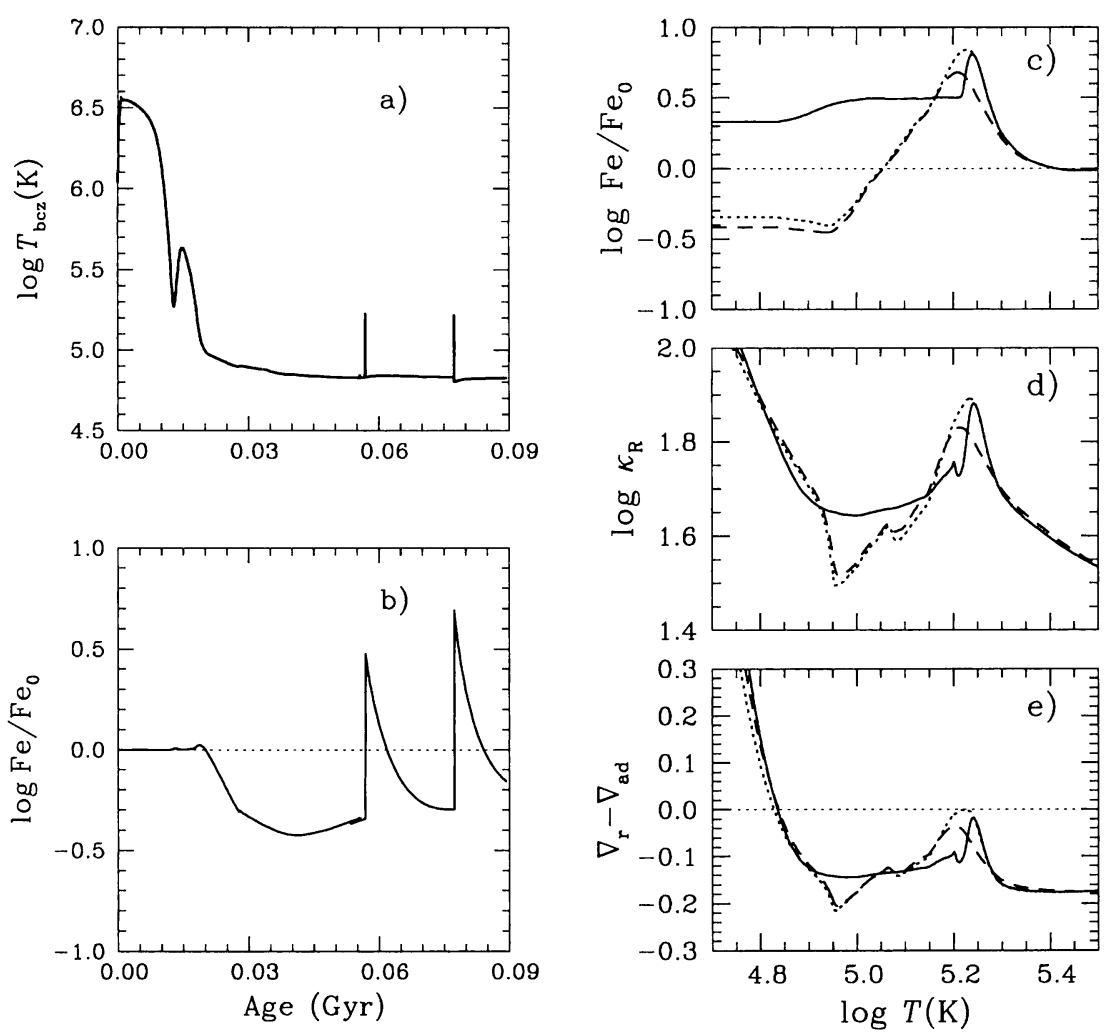

Figure 1. The figures $\mathbf{a}$ and $\mathbf{b}$ show respectively the evolution of the temperature at the bottom of the deepest surface convection $\left(T_{\mathrm{bcz}}\right)$ zone and of the surface Fe abundance in a $1.5 M_{\odot}$ model. The figures c, d, and e show at three ages (44 Myr: dashed line, 56.9 Myr: dotted line, and 57.9 Myr: solid line) the Fe abundance, the Rosseland mean opacity, and the difference between the radiative and adiabatic gradients as a function of temperature.

than $96 \%$ of the computing time (prior to parallelization) is spent on the atomic data.

Models have been computed from 1.0 to $4.0 M_{\odot}$ from the pre-main-sequence to the base of the giant branch. The solar model was used to calibrate the mixing length. More than 100 models have been computed up to now.

\subsection{Iron convection zones}

Probably the most striking difference between the basic models and standard stellar evolution models is the iron (or more properly iron-peak) convection zone they develop. Their natural appearance may be seen for instance in a $1.5 M_{\odot}$ model. On Figure $1 a$ and $1 b$ is shown the temperature at the bottom of the deepest surface convection $\left(T_{\mathrm{bcz}}\right)$ zone as well as the surface Fe abundance in this model for the first $90 \mathrm{Myr}$ of its evolution. At 57 and $76 \mathrm{Myr}$, spikes occur in the $T_{\mathrm{bcz}}$. It increases suddenly by slightly more than a factor of 2 . A third 
spike was about to occur at $90 \mathrm{Myr}$. The structural changes that occurred in the model may be seen by looking at Figure $1 c, 1 d$ and $1 e$. Between 44 and $56.9 \mathrm{Myr}$, a progressive increase of the Fe abundance occurred at a temperature of about $170000 \mathrm{~K}$. This occurs, without any arbitrariness, as a consequence of the temperature dependence of $g_{\mathrm{rad}}(\mathrm{Fe})$ which is larger than gravity for $T>$ $170000 \mathrm{~K}$ and becomes smaller below this temperature so that $\mathrm{Fe}$ is pushed from the deeper interior and accumulates around $170000 \mathrm{~K}$. The Rosseland mean opacity follows the trend of the $\mathrm{Fe}$ abundance while the difference between the radiative and adiabatic temperature gradients reaches zero at that location at 56.9 Myr. An Fe convection zone then appears briefly. Complete mixing of the mass above the Fe convection zone was then imposed. This is partly justified by the results of Latour et al. (1981) who showed that between superficial hydrogen and helium convection zones the mixing is essentially complete. This will be seen below to lead to a simple model for the AmFm stars. The imposed mixing forced redistribution of the Fe accumulated at $T=170000 \mathrm{~K}$ up to the stellar atmosphere region thus reducing its abundance and the Rosseland opacity at $170000 \mathrm{~K}$; the $\mathrm{Fe}$ convection zone consequently disappears. The zone is now stable and Fe starts to accumulate again below the shallower convection zone. This process repeats itself until enough $\mathrm{Fe}$ has accumulated for the Fe convection zone to remain in spite of the mixing with the surface. As can be seen in Figure $1 b$, each mixing event causes the surface iron abundance to increase suddenly, then to relax exponentially back to a slowly increasing baseline level.

Alternatively, one could choose to impose no mixing above the Fe convection zone. It then remains and grows once it has formed. In either case one has an Fe convection zone; its existence is not dependent on the mixing between the convection zones. While the no mixing assumption might be more appropriate the first time the Fe convection zone appears, our assumption of complete mixing above the Fe convection zone appears more appropriate when it is fully developed. It is supported by the accuracy with which the observed abundances are reproduced in the AmFm stars.

\section{The AmFm stars}

It appears compatible with the statistics of stellar rotation that all non magnetic A and early F stars $\left(7000<T_{\text {eff }}<10000 \mathrm{~K}\right.$ ) be Am or Fm stars (Abt \& Morrell 1995). The AmFm phenomenon is consequently not a fringe phenomenon. Its appearance must be the consequence of the normal stellar evolution of slowly rotating stars. The brightest star in the sky, Sirius A, is an Am star.

The basic model leads, with no arbitrary parameter, to abundance anomaly patterns that are very similar to those observed in AmFm stars. However, since the predicted anomalies are generally more important than the observed ones, a simple turbulent transport coefficient was introduced (Richer et al. 2000) to determine the properties of turbulent transport that can reduce these predictions to the observed levels:

$$
D_{\mathrm{T}}=\omega D(\mathrm{He})_{0}\left(\frac{\rho_{0}}{\rho}\right)^{n}
$$

where $n=2,3$ or 4 , and $D(\mathrm{He})_{0}$ is the atomic diffusion coefficient of He at density $\rho_{0}$. The turbulent diffusion coefficient is then equal to $\omega$ times the atomic 
diffusion coefficient of $\mathrm{He}$ at the reference density in the model of interest. It decreases inward as $\rho$ increases with an exponent that was varied between 2 and 4. It was found that the value of the exponent had little importance, so long as it was larger than, or equal to about 2. For AmFm stars, what matters is the mass which is mixed by turbulence. It is approximately given, in our models, by the mass above the point where $D_{\mathrm{T}}=2 D(\mathrm{He})$.

A detailed comparison of recent abundance determinations on Sirius to surface abundances calculated with various turbulence mixing is shown in Figure 18 of Richer et al. (2000). Five different groups of astronomers have, in eight papers, studied the surface chemical abundances of Sirius over the last seventeen years. When an element has been studied by different groups there appear variations in the determined abundance. Generally all groups agree as to the overor underabundance of an element but the size of the determined anomaly varies often significantly. Of the 28 species we calculate, the abundances of 16 have been determined. Since our models depend on only one parameter, the mass of the mixed zone, reproducing the abundance anomalies of all measured species is a very severe test. The models used to compare with Sirius all have an age of $250 \mathrm{Myr}$, compatible with the $T_{\text {eff }}$ of Sirius B and reasonable assumptions as to the original parameters of the binary system. The models differ by the size of the mixed zone which goes, for the models on Figure 18 of Richer et al. (2000), from $\Delta M / M_{*} \sim 3 \times 10^{-6}$ to $\Delta M / M_{*} \sim 1 \times 10^{-5}$. The model passes the test of the comparison very well. Of the 16 abundances, 12 are well reproduced by the model, 3 are not so well reproduced and one is a very uncertain observation. The model was also used to compare with AmFm stars in clusters. These have the advantage that their age is better known and that it is possible to get a handle on the original abundances through the abundances observed on late $\mathrm{F}$ or $\mathrm{G}$ stars. In Richer et al. (2000) comparisons were made to stars of the Hyades, the Pleiades, and Preasepe, with as much success as in Sirius except that the test is less rigorous since the abundances of fewer elements were observed.

We do not wish to claim that atomic diffusion coupled to turbulent transport are the only contributors to the AmFm phenomenon. However the accuracy of the observations will have to be improved before it is justified to look for the role of other processes. Such observations should now become possible in galactic clusters.

\section{LiBeB abundances and turbulence}

\subsection{Radiative accelerations of lithium}

$\mathrm{LiBeB}$ are not in the OPAL data since they do not contribute sufficiently to the Rosseland mean opacity. For the basic models their radiative accelerations were calculated in a consistent manner with the spectra of OPAL, including also the various corrections determined by Richer et al. (1997). The atomic data needed to calculate them is available and details are given in Richer et al. (in preparation). Figure 2 shows OPAL spectra of Fe and of the total opacity along with the Li spectrum. The frequency interval covers about one third of the total frequency range of the OPAL spectra. The most important Li line at that temperature is at $u \sim 6.7$, where the total opacity is dominated by a large number of $\mathrm{Fe}$ lines. 


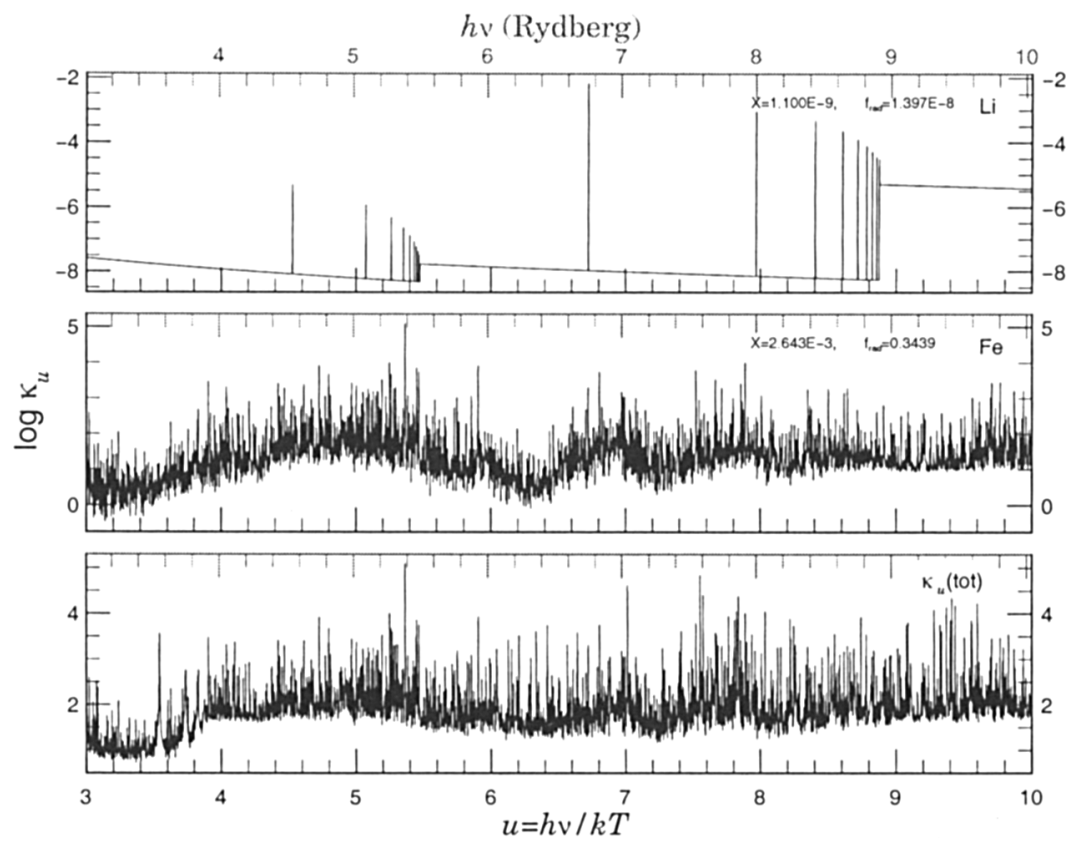

Figure 2. Iron OPAL spectra and the total OPAL opacity (in $\left.\mathrm{cm}^{2} \mathrm{~g}^{-1}\right)$ at a point in a stellar model where $\log T=5.2$ and $\log \rho=$ -5.4 ; the bulk of the radiative flux goes through this $u$ range. Also shown in the top panel is the Li absorption spectrum calculated at the same resolution. The mass fraction of $\mathrm{Li}$ and $\mathrm{Fe}$ are indicated, as well as the fraction of the total radiative flux each one absorbs.

The effect of taking into detailed account the competition for photons by the 28 species is shown on Figure 3. The Li radiative acceleration is shown there at four time steps along with the Fe abundance. While at the beginning of the evolution, the radiative acceleration on $\mathrm{Li}$ is greater than gravity over a certain mass interval, it becomes rapidly smaller everywhere as a consequence of the increase of the $\mathrm{Fe}$ abundance which reduces the flux of photons available to $\mathrm{Li}$. Furthermore the maximum of the radiative acceleration turns out to be in the Fe convection zone so that it would not influence transport there.

The use of models in which metals diffuse in a self-consistent manner strongly modifies the atomic diffusion of $\mathrm{Li}$. The radiative accelerations of $\mathrm{Li}$ differ in important ways from those of Richer \& Michaud (1993). Similar effects occur for $\mathrm{Be}$ and $\mathrm{B}$ whose radiative accelerations are shown in Richer et al. (in preparation).

\section{2. $\mathrm{Li}$ and $\mathrm{Be}$ as constraints on turbulence}

In Figure 4 is shown, at the age of the Hyades, the abundance of $\mathrm{Li}$ at the surface of stars between 5500 and $8600 \mathrm{~K}$. Small open circles and triangles are respectively the observed abundances and upper limits in the Hyades (Boesgaard \& 


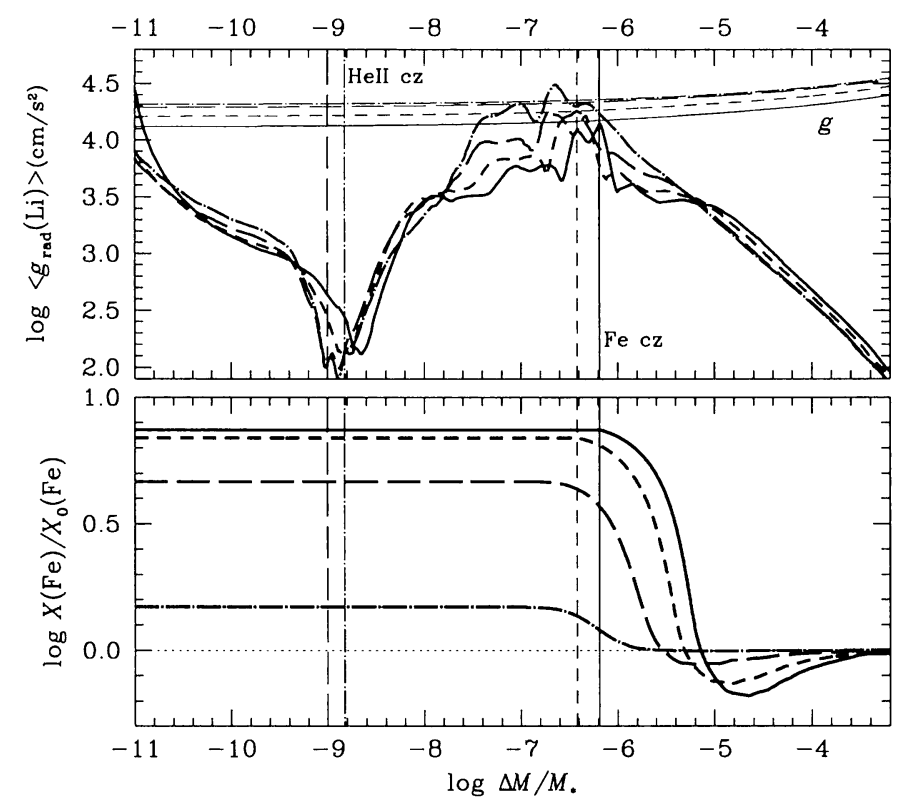

Figure 3. Lithium radiative acceleration (thick lines in the upper graph) and $\mathrm{Fe}$ abundance as a function of mass integrated from the surface, at different ages (10 Myr: dot-dashed lines, $70 \mathrm{Myr}$ : long dashed lines, 232 Myr: short dashed lines, and 411 Myr: solid lines), in a $2 M_{\odot}$ model with weak turbulence $(\omega=50, n=3)$. Gravity is shown in the upper graph as thin lines for each age. The vertical thin lines in both graphs show the depth of the surface convective zone.

Tripicco 1986; Boesgaard \& Budge 1988; Thorburn et al. 1993). The calculated models are identified on the figure.

The solid line links the models calculated with a mixing that reproduces approximately the surface abundances of AmFm stars. The same turbulent diffusion coefficient was used for all masses; no effort was made at optimizing the agreement for individual stars. The agreement for the five main-sequence AmFm stars of the Hyades (the five open circles between 8200 and $7200 \mathrm{~K}$ ) is quite satisfactory. The reduction of the Li abundance is by a factor of 2-3 at the age of the Hyades. The reduction factor is the same down to $6700 \mathrm{~K}$ and it progressively approaches 1.0 at $6500 \mathrm{~K}$. Overabundances of metals accompany the reduction of the Li abundance in these models (see Figs. 19 to 21 of Richer et al. 2000). While some of the stars of the Li gap (those where Li is underabundant by a factor of about 3) may have been affected by the same process that leads to the AmFm stars, this does not seem to be possible for all Li gap stars since the underabundances of $\mathrm{Li}$ remain small in these models. Furthermore, the accompanying overabundances of metals do not appear to be present in all $\mathrm{Li}$ gap stars.

The dashed line links models with no mixing beyond that occurring in convection zones. The only transport process is atomic diffusion. It is seen to 


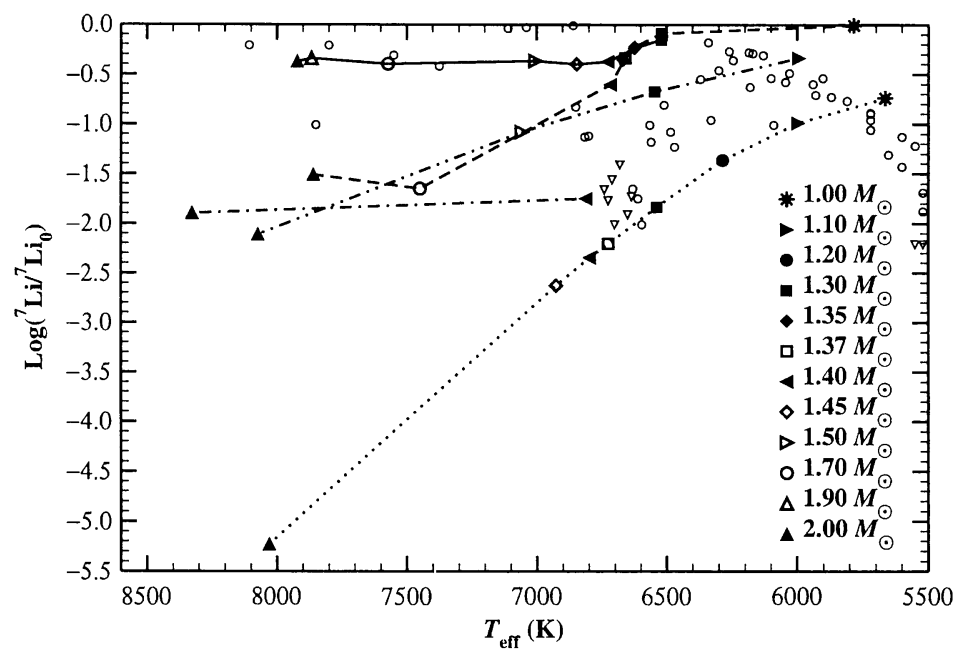

Figure 4. Surface lithium variation at the age of the Hyades. The small open circles and triangles represent respectively the measured values and the upper limits on lithium abundance. The solid line links models calculated with a mixing that reproduces approximately the surface abundances of the AmFm stars. The dashed line links models without turbulence where diffusion takes place below the Fe convection zone. The dotted line links models that were calculated with $n=2.5$ and a large enough turbulence to destroy lithium by more than a factor of 100 at the bottom of the Li gap of the Hyades. The dashed-double dotted line links models calculated with the same $n=2.5$ exponent as the models for the dotted line but with $\omega$ reduced by a factor 4 . The dot-dashed line links models calculated with a $n=0.72$ exponent that reproduces $\mathrm{Li}$ destruction in giant stars.

lead to much larger underabundance factors for $\mathrm{Li}$ than the model appropriate for the AmFm stars. The star at $T_{\text {eff }}=7900 \mathrm{~K}$ with a $1 / 10 \mathrm{Li}$ underabundance may have less turbulence than other AmFm stars and so a Li abundance closer to the no-turbulence result. The larger underabundance factors occur at higher $T_{\text {eff }}$ than the observed Li gap. It is also at a $T_{\text {eff }}$ higher by $800 \mathrm{~K}$ than the bottom of the Li gap obtained by Richer \& Michaud 1993. This shift is caused by Fe convection zones: they increase the depth of the total surface convection zone at a given $T_{\text {eff }}$. Because of the increased depth of the convection zone, $\mathrm{Li}$ is underabundant only by a factor of 3 at $T_{\text {eff }}=6700 \mathrm{~K}$ (the $1.4 M_{\odot}$ model). It is only in the more massive models that the mass in and above the Fe convection zone is small enough to allow underabundances by a factor of 30 at the age of the Hyades (in the $1.70 M_{\odot}$ star). That there should appear large underabundances of $\mathrm{Li}$ in this model is also related to the reduction of $g_{\mathrm{rad}}(\mathrm{Li})$ caused by the increased $\mathrm{Fe}$ abundance as described above.

The dotted line links models that were calculated with $n=2.5$ (see Eq. 2) and a large enough value of $\omega$ to destroy lithium by slightly more than a factor of 100 at the $T_{\text {eff }}$ where the Li gap of the Hyades is deepest. It reproduces 


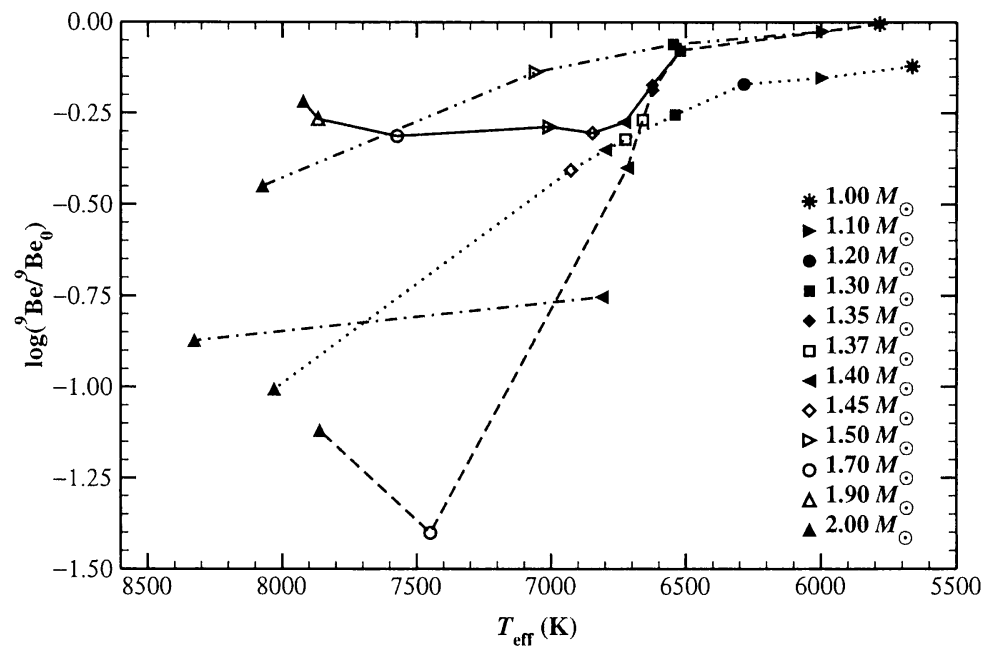

Figure 5. Surface beryllium variation at the age of the Hyades. The same convention as in Figure 4 is used for the lines.

approximately the shape of the cool side of the Li gap. As one considers lower $T_{\text {eff }}$ stars, the distance between the bottom of the convection zone and the $\mathrm{Li}$ burning area is reduced favoring $\mathrm{Li}$ destruction but the mass of the surface $\mathrm{Li}$ reservoir increases more rapidly than the net destruction rate and thus the $\mathrm{Li}$ surface anomaly is reduced. However this model still destroys Li by far too large a factor in normal stars hotter than the Li gap $\left(T_{\text {eff }}>6700 \mathrm{~K}\right)$. The dashed-double dotted line links models calculated with the same exponent as the models used to calculate the dotted line, but with a reduced factor $\omega$. It allows to evaluate the range of acceptable $\omega$ values.

The dot-dashed line links models calculated with a $n=0.72$ exponent that reproduces the Li destruction presumably occurring on the main sequence but observed in giant stars (Talon et al., in preparation). It is seen to lead to more destruction than observed in the hotter stars of the Hyades.

A similar figure is shown for Be (Fig. 5). No Be abundance have been determined in Hyades stars. The field Be abundances are discussed elsewhere in this conference by Deliyannis (see also Deliyannis et al. 1998). The solar Be abundance is discussed by Balachandran. Since the Be superficial abundance is usually much closer to the original abundance than $\mathrm{Li}$, this implies a much smaller destruction for $\mathrm{Be}$ than for Li. In turn this implies a lower limit for $n$ that is probably larger than $n=0.72$. The extent to which the field star $\mathrm{Li}$ and Be abundances put limits on $n$ will be further discussed in Richer et al. (in preparation).

\section{Constraints on turbulence}

The preceding discussions establish a number of constraints that turbulent transport must satisfy: 1) Abundance anomalies in AmFm stars (slowly rotating A 
and F stars) imply mixing of the outer $\sim 10^{-5}$ of the star's mass. 2) The existence of "normal" $\mathrm{A}$ and $\mathrm{F}$ stars (more rapidly rotating $\mathrm{A}$ and $\mathrm{F}$ stars) requires mixing of at least $3 \times 10^{-4}$ to $10^{-3}$ of the star's mass if the anomalies are not to exceed $0.1 \mathrm{dex}$ and of not more than $10^{-2}$ if $\mathrm{Li}$ is not to be completely destroyed. 3) The Li gap implies partial mixing of the outer $10^{-2}$ of the mass since that is the depth where $\mathrm{Li}$ is destroyed. This mixing is probably not complete however since turbulence has to lead to less Li destruction on the cool side of the $\mathrm{Li}$ gap. If the mixing were complete, no $\mathrm{Li}$ would remain. 4) While $\mathrm{Be}$ is less destroyed than $\mathrm{Li}$, it is partly destroyed. The partial $\mathrm{Li}$ and $\mathrm{Be}$ destruction imply a density dependence of turbulence $(n \gtrsim 2$; see Eq. 2$)$. The turbulent transport close to the region of $\mathrm{Li}$ burning is also constrained by observations of $\mathrm{Li}$ abundance in giant stars as described at this conference by Charbonnel. 6) The sun requires similar turbulence as required for $\mathrm{Li}$ and Be destruction in F stars. This turbulence is weak enough for the gravitational settling of He to lead to a $10 \%$ reduction of the He abundance in the solar convection zone.

These constraints can now be improved upon by more accurate abundance determinations in cluster stars. This is within reach of the new generation of large telescopes. Stellar evolution modeling also needs to be improved. The results presented here show that it is within reach, using available computers and atomic data bases.

\section{References}

Abt, H. A., \& Morrell, N. I. 1995, ApJS, 99, 135

Boesgaard, A. M., \& Budge, K. G. 1988, ApJ, 332, 410

Boesgaard, A. M., \& Tripicco, M. J. 1986, ApJ, 302, L49

Burgers, J. M. 1969, Flow equations for composite gases, (New York: Academic Press)

Chaboyer, B., Demarque, P., \& Pinsonneault, M. H. 1995, ApJ, 441, 865

Deliyannis, C. P., Boesgaard, A. M., Stephens, A., King, J. R., Vogt, S. S., \& Keane, M. J. 1998, ApJ, 498, L147

Latour, J., Toomre, J., \& Zahn, J.-P. 1981, ApJ, 248, 1081

Proffitt, C. R., \& Michaud, G. 1991, ApJ, 380, 238

Richard, O., Vauclair, S., Charbonnel, C., \& Dziembowski, W. A. 1996, A\&A, 312,1000

Richer, J., \& Michaud, G. 1993, ApJ, 416, 312

Richer, J., Michaud, G., \& Massacrier, G. 1997, A\&A, 317, 968

Richer, J., Michaud, G., Rogers, F. J., Iglesias, C. A., Turcotte, S., \& LeBlanc, F. 1998, ApJ, 492, 833

Richer, J., Michaud, G., \& Turcotte, S., 2000, ApJ, in press

Thorburn, J. A., Hobbs, L. M., Deliyannis, C. P., \& Pinsonneault, M. H. 1993, ApJ, 415, 150

Turcotte, S., Richer, J., Michaud, G., Iglesias, C. A., \& Rogers, F. J. 1998, ApJ, 504,539

Vauclair, S. 1988, ApJ, 335, 971 